\title{
Transient intraneuronal $A \beta$ rather than extracellular plaque pathology correlates with neuron loss in the frontal cortex of APP/PS1KI mice
}

\author{
Ditte Zerlang Christensen - Sophie Luise Kraus • \\ Antonius Flohr · Marie-Caroline Cotel • \\ Oliver Wirths - Thomas A. Bayer
}

Received: 1 October 2008/Revised: 22 October 2008/Accepted: 22 October 2008/Published online: 31 October 2008

(C) The Author(s) 2008. This article is published with open access at Springerlink.com

\begin{abstract}
The accumulation of beta-amyloid (A $\beta)$ plaques and neurofibrillary tangles consisting of hyperphosphorylated tau protein are pathological features of Alzheimer's disease (AD) commonly modeled in mice using known human familial mutations; however, the loss of neurons also found to occur in $\mathrm{AD}$ is rarely observed in such models. The mechanism of neuron degeneration remains unclear but is of great interest as it is very likely an important factor for the onset of adverse memory deficits occurring in individuals with $\mathrm{AD}$. The role of $\mathrm{A} \beta$ in the neuronal degeneration is a matter of controversial debates. In the present study we investigated the impact of extracellular plaque $A \beta$ versus intraneuronal $A \beta$ on neuronal cell death. The thalamus and the frontal cortex of the APP/ PS1KI mouse model were chosen for stereological quantification representing regions with plaques only (thalamus) or plaques as well as intraneuronal $A \beta$ (frontal cortex). A loss of neurons was found in the frontal cortex at the age of 6 months coinciding with the decrease of intraneuronal immunoreactivity, suggesting that the neurons with early intraneuronal $A \beta$ accumulation were lost. Strikingly, no neuron loss was observed in the thalamus despite the development of abundant plaque pathology with levels comparable to the frontal cortex. This study suggests that
\end{abstract}

O. Wirths and T. A. Bayer have equally contributed to this work.

D. Z. Christensen · S. L. Kraus · A. Flohr · M.-C. Cotel ·

O. Wirths $(\square) \cdot$ T. A. Bayer

Division of Molecular Psychiatry

and Alzheimer Ph.D. Graduate School,

Department of Psychiatry, University of Goettingen,

von-Siebold-Str. 5, 37075 Göttingen, Germany

e-mail: owirths@uni-goettingen.de

T. A. Bayer

e-mail: tbayer@gwdg.de plaques have no effect on neuron death whereas accumulation of intraneuronal $A \beta$ may be an early transient pathological event leading to neuron loss in $\mathrm{AD}$.

Keywords Transgenic mice - Amyloid . Intracellular $\mathrm{A} \beta \cdot$ Intraneuronal $\mathrm{A} \beta$. Alzheimer . Neuron loss

\section{Introduction}

Alzheimer's disease (AD) is pathologically characterized by the deposition of beta-amyloid $(A \beta)$ plaques and neurofibrillary tangles consisting of hyperphosphorylated Tau protein. A further pathological determinant is loss of neurons, which occurs predominantly in the cortex, the hippocampal formation, and the nucleus basalis of Meynert $[2,3,18,22,25]$. Whereas accumulations of $\mathrm{A} \beta$ plaques and Tau hyperphosphorylation are reflected in transgenic mice expressing either mutant human APP or Tau, loss of neurons is only rarely observed in these mouse models. Unlike $A \beta$ and Tau pathology, neuron loss is not present in pre-clinical $\mathrm{AD}$ and thus may be responsible for the onset of the adverse symptoms occurring with the transition to $\mathrm{AD}$ [23]. The underlying mechanism of neuronal loss is therefore of great interest.

Plaques are known to cause disturbances in the fiber network developing dystrophic swellings of surrounding fibers and mediate loss of dendritic spines $[1,7,19,29]$. In addition, plaques have been shown to decrease synaptic bouton density, preferably of cholinergic cortical fibers [13]; however, the direct functional effects of these fiber disturbances and their effects on neuronal survival are unknown. Besides extracellular plaque pathology, intraneuronal accumulation of $A \beta$ peptides has been repeatedly 
reported in $\mathrm{AD}[6,9,11]$, as well as in Down syndrome patients $[12,15]$ and is believed to precede extracellular plaque deposition.

The APP/PS1KI mice represent a valuable model system, as they develop an extensive neuron loss in the CA1 region of the hippocampal formation [4], a characteristic also found in $\mathrm{AD}$ patients [22], which is preceded by strong intraneuronal $\mathrm{A} \beta$ accumulation, as well as a severe motor pathology, axonal degeneration marked by dystrophic fibers, and behavioral deficits already at the age of 6 months [26, 29]. In addition, a correlation between accumulation of intraneuronal $A \beta$ and neuron loss in the cholinergic system of these mice has been recently established [5]. The present study was designed specifically to investigate the impact of plaques versus intraneuronal $A \beta$ on neuronal survival by stereological quantification of neuron numbers in the thalamus and frontal cortex, representing regions with plaques only or additional intraneuronal $\mathrm{A} \beta$ accumulations, respectively.

\section{Materials and methods}

\section{Transgenic mice}

The generation of APP/PS1KI mice has been described previously [4]. In brief, human mutant APP751 containing the Swedish and London mutations is overexpressed under the control of the murine Thy-1 promoter, whereas murine PS1 with the M233T and L235P FAD-linked mutations is expressed under the control of the endogenous mouse PS1 promoter. All mice designated PS1KI were homozygous for PS1 knock-in mutations, in comparison to the APP/ PS1KI mice that had one additional hemizygous APP751SL transgene. APP/PS1KI mice were a generous gift of Dr. Laurent Pradier, Sanofi-Aventis, France. All animals were handled according to German guidelines for animal care.

Frozen tissue processing and cresyl violet-staining

Stereological quantification was performed on perfused, frozen, cresyl-violet stained sections. For this purpose mice were anesthetized and transcardially perfused with ice-cold phosphate buffered saline (PBS) followed by $4 \%$ (w/v) paraformaldehyde (PFA) dissolved in PBS. Brains were carefully removed, divided at the midline, and post-fixated in $4 \%$ PFA for $2 \mathrm{~h}$. After cryo-protection in $30 \%$ sucrose in PBS overnight, the brains were quickly frozen and stored at $-80^{\circ} \mathrm{C}$ until further processing. The brains were systematically cut on a cryostat in ten series of $30 \mu \mathrm{m}$ coronal sections. All sections were stored frozen at $-80^{\circ} \mathrm{C}$ until further processing. One series comprising every tenth section from each animal was carefully mounted in PBS onto super frost glass slides. When dried, all the slices were stained with cresyl violet. Briefly, the sections were delipidated in a $1 \mathrm{M}$ natrium acetate $/ 0.15 \mathrm{M}$ acetic acid solution followed by a wash in $2 \%$ Triton X-100 in ethanol, and finally stained in $0.01 \%$ cresyl-violet solution.

\section{Stereology}

Stereological neuron quantification was performed using design-based stereology on $30 \mu \mathrm{m}$ cresyl-violet stained frozen hemisphere sections using StereoInvestigator 7 (MicroBrightfield) and a BX51 microscope (Olympus). Regions for quantification were defined as visualized in Fig. 5a. The frontal cortex was quantified from Bregma $3.08-0.26$ [16] owing to the accumulation of intraneuronal $\mathrm{A} \beta$. The thalamus was quantified from Bregma -0.94 to -2.54 [16] owing to the tremendous amount of plaque accumulation, however, without any accumulation of intraneuronal $A \beta$. Only female mice were analyzed at the ages of $2(n=5), 6(n=6)$ and 12 months $(n=8)$. The optical fractionator method was applied counting every tenth section resulting in coefficient of errors $<0.06$. Estimation of the total number of neurons in hemispheres was performed according to the principle of stereology $[17,21$, 24]. Counting was performed by an investigator blinded to the genotype of the animals.

\section{Immunohistochemistry on paraffin sections}

Mice were transcardially perfused with 4\% PFA in PBS and brains were carefully dissected. Post-fixation was carried out in $4 \%$ buffered formalin at $4{ }^{\circ} \mathrm{C}$ before the tissue was embedded in paraffin. Immunohistochemistry was performed on $4-\mu \mathrm{m}$ sagittal paraffin sections, as described previously [28]. In brief, sections were deparaffinized in xylene and rehydrated in a series of ethanol. After treatment with $0.3 \% \mathrm{H}_{2} \mathrm{O}_{2}$ in PBS to block endogenous peroxidases, antigen retrieval was achieved by boiling sections in $0.01 \mathrm{M}$ citrate buffer $\mathrm{pH} 6.0$, followed by 3 min incubation in $88 \%$ formic acid. Non-specific binding sites were blocked by treatment with skim milk and fetal calf serum in PBS, prior to the addition of the primary antibodies. The following antibodies were used: 23850 against human APP (generously provided by Gerd Multhaup), an N-terminal $A \beta$ antibody (A $\beta[\mathrm{N}]$, IBL, Hamburg, Germany), an antibody detecting the aspartate at position 1 (N1D) and an antibody against pyroglutamate at position $3(\mathrm{~N} 3 \mathrm{pE}$; both antibodies were a generous gift from Takaomi C. Saido), G2-10 detecting A $\beta 40$ (The Genetics Company), as well as 4G8 (Covance). Primary antibodies were incubated overnight in a humid chamber at room temperature followed by incubation with biotinylated secondary antibodies (DAKO, 
Glostrup, DK). Staining was visualized using the ABC method with Vectastain kit (Vector Laboratories, Burlingame, USA) and diaminobenzidine as chromogen providing a reddish brown color. Counterstaining was carried out with hematoxylin. PS1KI control mice were consistently negative for APP and $A \beta$ staining.

\section{Quantification of $\mathrm{A} \beta$ load}

For each animal, five paraffin embedded sections, which were at least $25 \mu \mathrm{m}$ afar from each other, were stained simultaneously with DAB as chromogen and using a low concentration of the $4 \mathrm{G} 8$ antibody $(1: 10,000)$ to minimize any cross-reaction with APP. The relative A $\beta$ load was evaluated in the frontal cortex and the thalamus using an Olympus BX-51 microscope equipped with an Olympus DP-50 camera and the ImageJ software (NIH, USA). Representative pictures of $10 \times$ magnification were systematically captured in each region. Using ImageJ the pictures were binarized to 8-bit black and white pictures and a fixed intensity threshold was applied defining the DAB staining. Measurements were performed for a percentaged area covered by 4G8 DAB staining, as well as for the number of grains per $\mathrm{mm}^{2}$ and the average size of the grains. In the thalamus where no intraneuronal $\mathrm{A} \beta$ was found, the grains correspond to plaques, whereas the grains in the frontal cortex include intraneuronal deposits as well.

\section{Statistical analysis}

Stereological quantifications were analyzed using two-way ANOVA followed by Bonferroni posttests. All data given apply to hemispheres. The A $\beta$ pathology was analyzed using one-way ANOVA followed by $t$ tests. Data are presented as mean \pm SEM. Significance levels were given as follows: $* * * P<0.001 ; * * P<0.01 ; * P<0.05$. All statistical analysis was performed using GraphPad Prism version 4.03 for Windows (GraphPad Software, San Diego, CA, USA).

\section{Results}

$\mathrm{A} \beta$ accumulation in the frontal cortex and thalamus

Massive intra- and extracellular $\mathrm{A} \beta$ accumulations were observed in the frontal cortex by immunohistochemical staining using the $4 \mathrm{G} 8$ antibody recognizing a central epitope within the $A \beta$ peptide (Fig. $1 \mathrm{a}-\mathrm{h}$ ). The intraneuronal pathology of the frontal cortex is already very prominent at the age of 1.5 months where virtually no or very few plaques are visible (Fig. 1a, e). Already at 2 months, plaque pathology starts to develop at a greater degree; however, intraneuronal $A \beta$ is still abundantly present (Fig. $1 \mathrm{~b}, \mathrm{f}$ ). At the age of 6 months plaque pathology has been dramatically developed; however, the staining of intracellular $\mathrm{A} \beta$ aggregates decreases and is no longer a dominating pathological feature (Fig. 1c, g). From 6 to 12 months, the pathology of the frontal cortex does not seem to change much, except that the plaques may become more densely packed (Fig. 1d, h). The thalamus is found to develop only plaques and no intraneuronal aggregates (Fig. 1i-p). The pathology of this region starts later than in the cortex at about the age of 2 months, therefore having no pathology at 1.5 months (Fig. 1i, m). From 2 to 6 months, numerous plaques develop and the pathology even increases further till the age of 12 months (Fig. $1 j-1, n-p$ ).

As the 4G8 antibody has been shown to weakly cross react with APP in Western blots, the antibody was highly diluted $(1: 10,000)$ in order to recognize only $A \beta$. Using a variety of different dilutions, this concentration has been identified to show no staining of APP (not shown). The presence of intracellular $A \beta$ aggregates was in addition confirmed by immunostaining with an $\mathrm{N}$-terminal specific $A \beta$ antibody $(A \beta N)$. Again, prominent accumulation of intraneuronal $A \beta$ staining was found in the frontal cortex already at the age of 1.5 and 2 months disappearing at 6 months (Fig. 2a-d). Furthermore, confirming the 4G8staining, no intraneuronal $\mathrm{A} \beta$ immunoreactivity was detected in the thalamus regardless of age (Fig. 2e-h). In addition, different end-specific $\mathrm{A} \beta$ antibodies have been used to demonstrate the specificity of intraneuronal $A \beta$ immunorectivity (Fig. 3e-g).

The regional difference in the accumulation of intraneuronal A $\beta$ seems to be a consequence of the APP transgene expression pattern, as the APP transgene is abundantly expressed in the pyramidal layers of the frontal cortex, whereas no expression is found in the thalamus (Fig. 3a-d).

To further characterize the development of $A \beta$ pathology over time in the two regions chosen for stereology, the A $\beta$ accumulation was quantified using the 4G8 antibody and a software density measurement technique (Fig. 4). At 2 months of age the area covered by $\mathrm{A} \beta$ deposits was already $4.7 \%$ in the frontal cortex owing to the massive accumulation of intraneuronal $\mathrm{A} \beta$, but only $1.5 \%$ in the thalamus because of the absence of intracellular $A \beta$ and the later onset of extracellular plaque pathology. At 6 months of age, the percentage of the area covered by $\mathrm{A} \beta$ deposits has increased to $12.1 \%$ in the frontal cortex and $8.5 \%$ in the thalamus. From the age of $6-12$ months, the A $\beta$-covered area remains stable in the frontal cortex, but increases further to $13.9 \%$ in the thalamus (Fig. 4a, b). Looking at average $A \beta$ grain size and grain numbers in each region, it is evident that the steady increase in the percentile $\mathrm{A} \beta$-covered area observed in the thalamus over time is solemnly due to a dramatic increase in plaque number, 

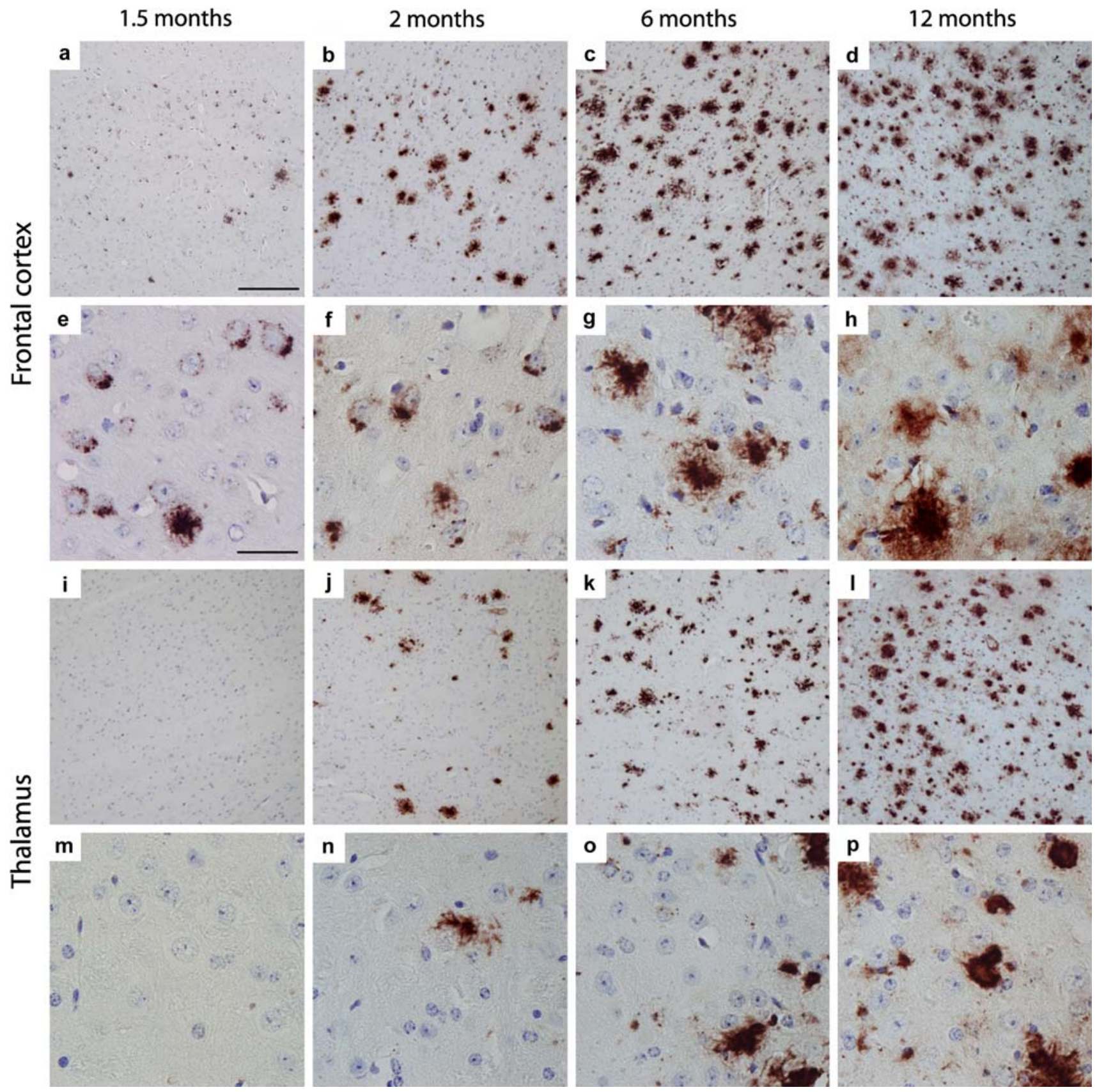

Fig. 1 Immunostaining of $A \beta$ peptides in frontal cortex $(\mathbf{a}-\mathbf{h})$ and thalamus (i-p) of 1.5-, 2-, 6-, and 12-month-old APP/PS1KI mice. Prominent accumulation of intraneuronal $A \beta$ is present in the frontal cortex already at 1.5 months with only very mild plaque pathology (a, e). More plaques are evident in the frontal cortex of 2-month-old mice where intraneuronal $A \beta$ is still highly abundant $(\mathbf{b}, \mathbf{f})$. The plaque pathology dramatically increases in the frontal cortex from 2 to 6 months of age with a concomitant decrease in intraneuronal $A \beta$ accumulation $(\mathbf{c}, \mathbf{g})$. The pathology seems mostly unchanged in 12-month-old mice, however, with a tendency of the plaques to become denser $(\mathbf{d}, \mathbf{h})$. No A $\beta$ pathology is observed in the thalamus of 1.5-month-old mice $(\mathbf{i}, \mathbf{m})$ which start to develop plaques at the age of 2 months, however, without any accumulation of intraneuronal A $\beta$ (j, n). As for the frontal cortex, the amount of plaques in the thalamus increased dramatically from 2- to 6-month-old mice $(\mathbf{k}, \mathbf{o})$ and even further to 12-month-old mice (l-p) (scale bar $\mathbf{a}-\mathbf{d}, \mathbf{i}-\mathbf{l}: 200 \mu \mathrm{m} ; \mathbf{e}-\mathbf{h}$, $\mathbf{m}-\mathbf{p}: 33 \mu \mathrm{m}$

age is also exclusively dependent on a 2.7 -fold increase in grain numbers as the average grain size is unchanged. However, in contrast to the thalamus, changes are seen in the composition of frontal cortical deposits, as the average 

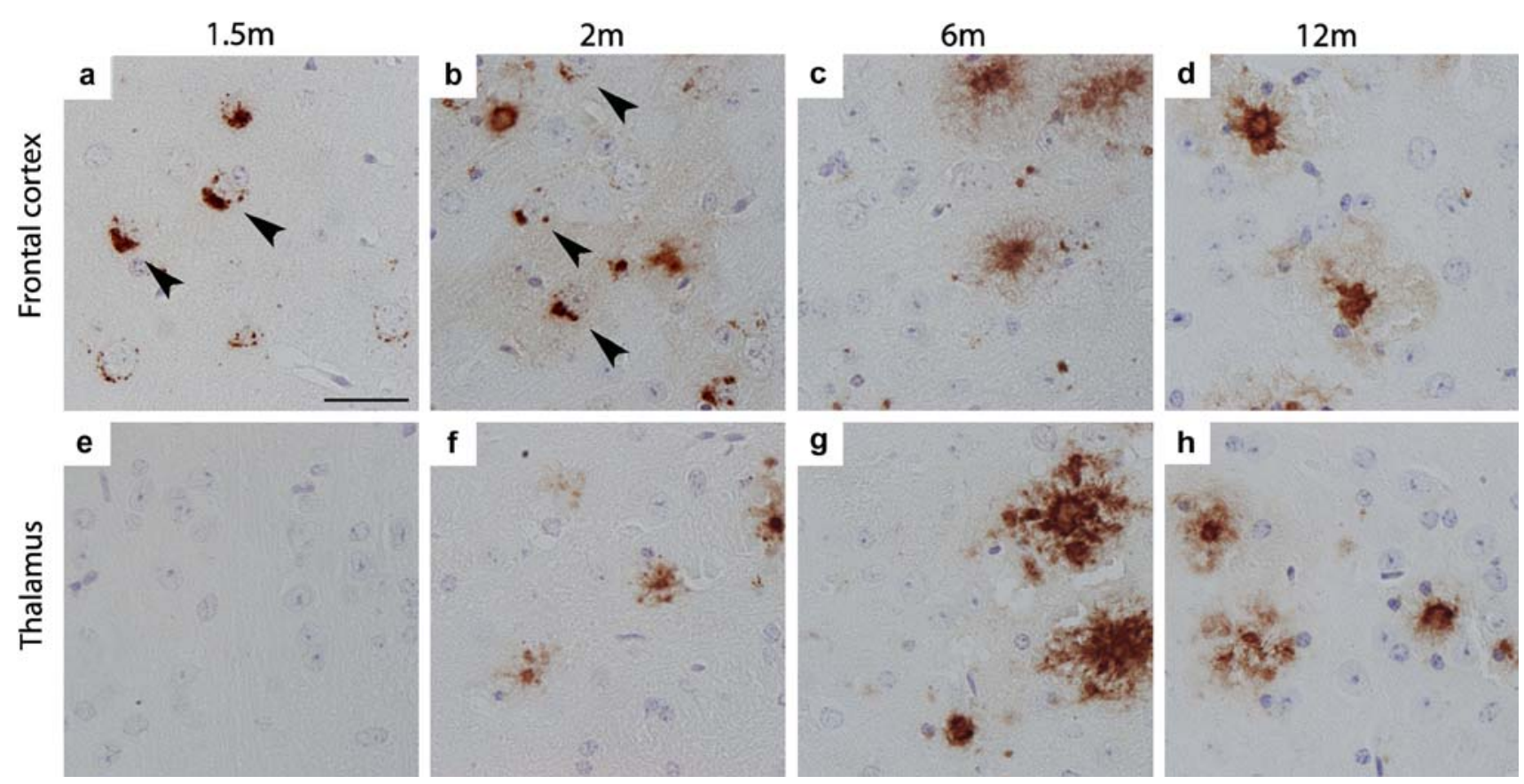

Fig. 2 The presence of intracellular $A \beta$ in the frontal cortex was confirmed by immunostaining of $A \beta$ peptide using $A \beta$ antibodies specifically binding the N-terminus. As with the 4G8 antibody, the intracellular $\mathrm{A} \beta$ staining was found to be prominent already at
1.5 month of age (a) as well as at 2 months (b), but disappearing at 6 and 12 months of age (c, d). No intraneuronal $A \beta$ staining was detected in the thalamus regardless of age (e-h) (scale bar $\mathbf{a}-\mathbf{h}$ : $33 \mu \mathrm{m}$
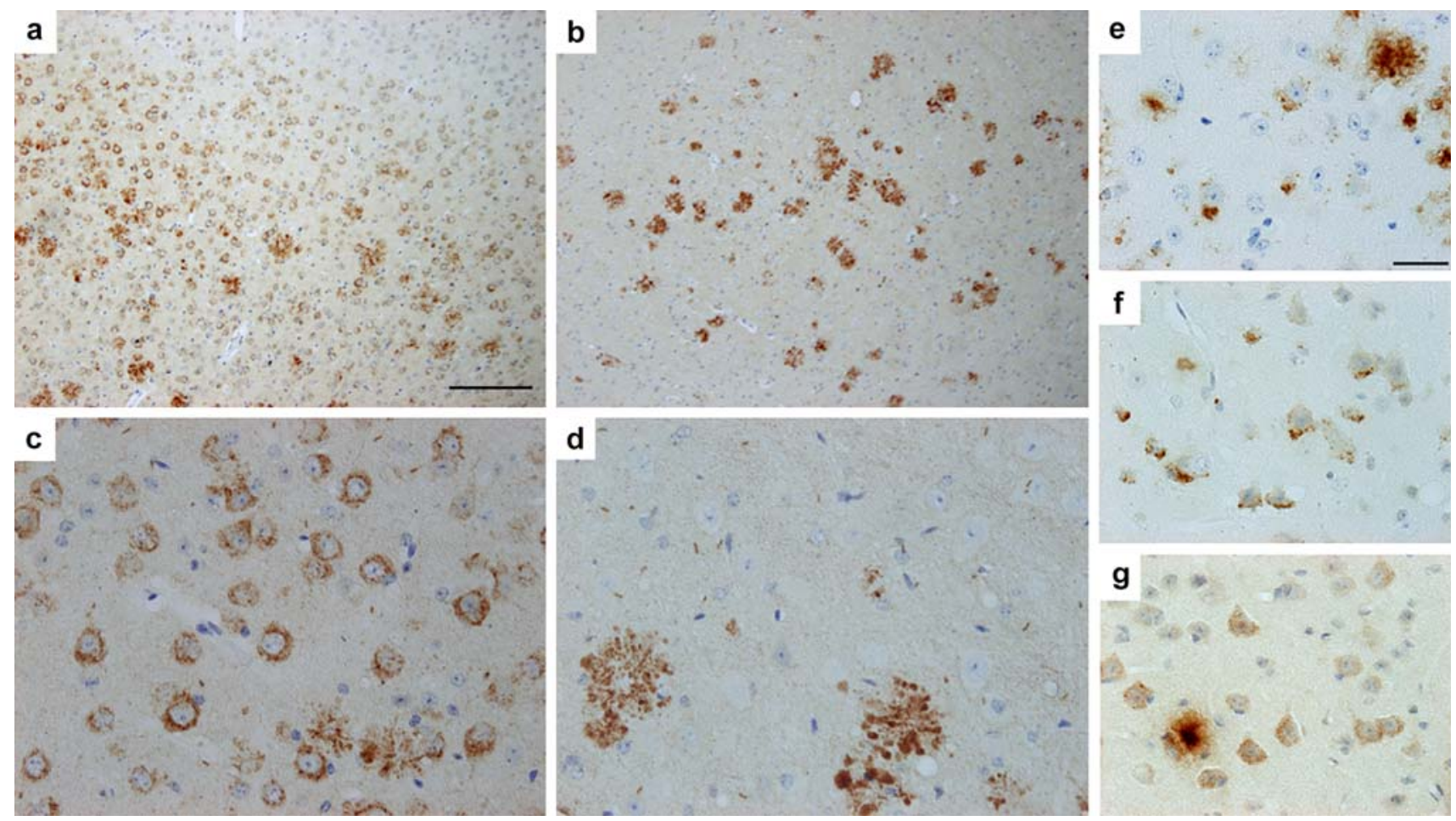

Fig. 3 Immunostaining of human APP in frontal cortex $(\mathbf{a}, \mathbf{c})$ and thalamus (b, d) of 2-month-old APP/PS1KI mice. The APP transgene is expressed in the neurons of the frontal cortex $(\mathbf{a}, \mathbf{c})$ but not of those in the thalamus, where only dystrophic fibers mostly surrounding plaques are stained (b, d). Endspecific antibodies to rule out APPcross-reactivity in the intraneuronal $\mathrm{A} \beta$ stainings. The following antibodies have been used: N1D (recognizing N-terminal A $\beta$ starting with aspartate at position $1 ; \mathbf{e}$ ), $\mathrm{N} 3 \mathrm{pE}$ (recognizing $\mathrm{N}$-terminal $\mathrm{A} \beta$ starting with pyroglutatmate at position $3 ; \mathbf{f}$ ) and the C-terminal specific antibody G2-10 recognizing A $\beta 40$ peptides (g) (scale bar: a, c: $200 \mu \mathrm{m} ; \mathbf{b}, \mathbf{d}: 50 \mu \mathrm{m} ; \mathbf{e}-\mathbf{g}: 33 \mu \mathrm{m}$ 
a
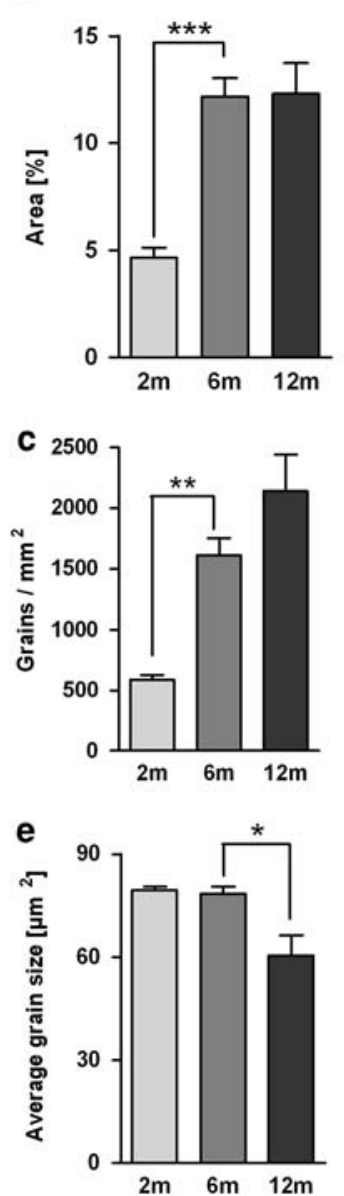

b
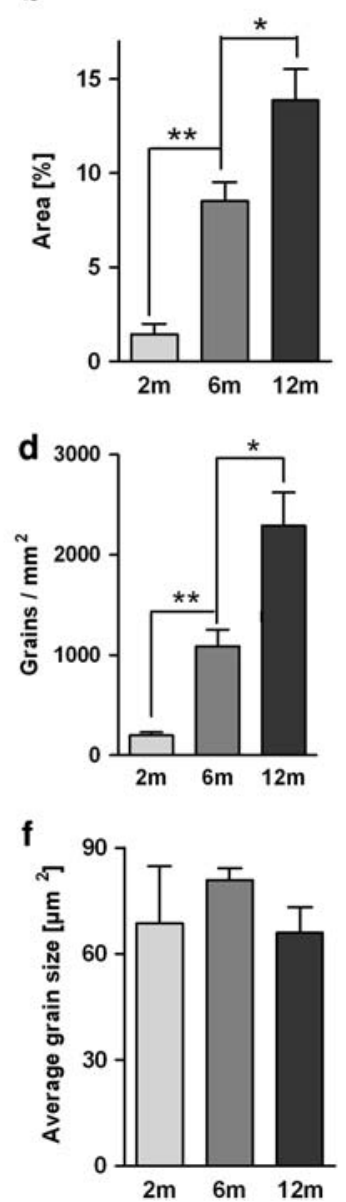

Fig. 4 Quantification of $\mathrm{A} \beta$ accumulation in frontal cortex and thalamus. About $5 \%$ of the frontal cortical area is covered by $\mathrm{A} \beta$ peptide in 2-month-old mice rising 2.6 times until the age of 6 months covering $12.1 \%$, with no further increase at 12 months of age (a). Only $1.5 \%$ of the thalamic area was covered by A $\beta$ peptide in the 2-month-old mice; however, this increased 5.8 times to $8.5 \%$ 6-month-old mice, resulting in $13.9 \%$ coverage at the age of 12 months (b). The increase of $A \beta$ load in the frontal cortex from the age of 2-6 months was due to an increased number of deposits (c) as no change in average deposit size was detected (e). Despite of unchanged levels in the frontal cortex between 6 and 12 months, a change in the composition of the deposits was seen as the average size decreased (c) and the number of deposits tended to increase $(\mathbf{e}, P=0.075)$. In the thalamus, the increase in $A \beta$ accumulation was observed to be solemnly due to increased number of deposits (d) as the average deposit size did not change with age (f). The number of animals analyzed was at 2 months: 3 ; 6 months: 5; 12 months: 4 . All error bars represent mean \pm SEM $* * * P<0.001 ; * * P<0.01 ; * P<0.05$

size decreases confirming the observation that the plaques become more dense, and the number of deposits tend to increase being close to significance $(P=0.075$; Fig. $4 \mathrm{c}, \mathrm{e})$.

In order to separate the effect of intracellular $A \beta$ accumulation on neuronal survival in the frontal cortex from the effect of plaques, we selected the thalamus as an appropriate region for comparison. Thus the amount of extracellular accumulating plaques in the thalamus is in a

comparable range to the $A \beta$ load observed in the frontal cortex, however, without any accumulation of intraneuronal $\mathrm{A} \beta$ peptides.

Stereology in the frontal cortex and thalamus

The counting areas of the frontal cortex and thalamus were defined according to Fig. 5a. A loss of neurons of 28 and $35 \%$ was found in the frontal cortex of the APP/PS1KI mice compared to PS1KI control mice at the age of 6 and 12 months, respectively (Fig. 5b). However, no significant difference was found between the neuron numbers of the frontal cortex at these ages in APP/PS1KI mice correlating with the finding of no further changes in $A \beta$ accumulation in the frontal cortex. Corresponding reductions in frontal cortical volumes were found at the age of both $6(25 \%)$ and $12(23 \%)$ months (Fig. 5d). In addition, an increase of $86 \%$ in frontal cortical volume was found from the age of 2 6 months in the PS1KI control mice which is due to normal growth in these young animals. A volume increase of 50\% was also identified for the APP/PS1KI frontal cortex from 2 to 6 months. This was less than found in the PS1K1 mice due to the volume loss observed at the age of 6 months in the bigenic mice.

Strikingly, no change was observed in the neuron numbers of the thalamus between 2-, 6-, and 12-month-old mice despite the development of massive plaque pathology (Fig. 5e). Correspondingly, no change in thalamic volume was found between APP/PS1KI and PS1KI control mice at any age investigated (Fig. 5d). As for the cortex, an increase in the thalamic volume of $75 \%$ was found for the PS1KI from the age of 2-6 months owing to the normal growth. This increase was $30 \%$ for the APP/PS1KI mice, however, only being close to significance $(P=0.072)$.

\section{Discussion}

A recent study from our laboratory reported a correlation between the accumulation of intracellular $A \beta$ and the loss of neurons in the cholinergic system of APP/PS1KI mice. Also, a dose effect was implied as one motor nucleus accumulated a larger amount of intraneuronal $A \beta$ and at an earlier time point as compared to another motor nucleus, and correspondently suffered a more extensive and earlier neuron loss [5]. This study strongly supported the hypothesis of intracellular $A \beta$ being a major trigger of neuron death in $\mathrm{AD}$, and the present study was designed to more specifically investigate the toxic effect of intracellular $A \beta$ versus extracellular plaque $A \beta$ on neuronal survival. For this reason, the present study focuses on two regions with distinct pathological differences regarding plaque development and intraneuronal $\mathrm{A} \beta$ aggregation. The 
a

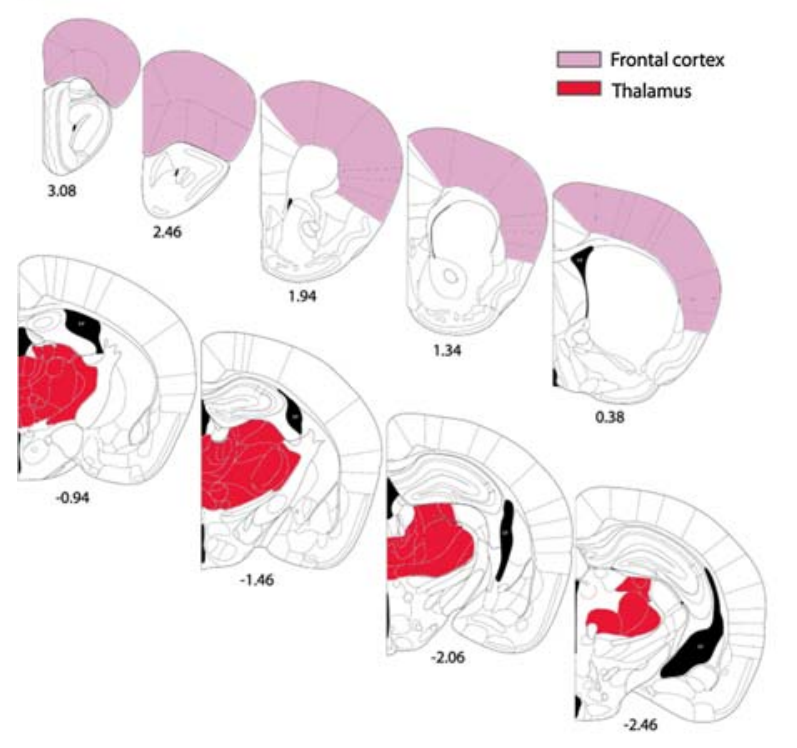

Fig. 5 Schematic presentation of counting areas and stereological quantification of neuron numbers. The frontal cortex was quantified from Bregma 3.08 to 0.26 and the thalamus was quantified from Bregma -0.94 to -2.54 (a). Figures were created using the mouse atlas by Paxinos and Franklin [16]. A significant neuron loss of 28 and $35 \%$ was found in the frontal cortex at the age of 6 and 12 months, respectively (b). Corresponding decreases were observed in frontal cortical volumes at both $6(25 \%)$ and $12(23 \%)$ months in APP/PS1KI mice. In addition, a volume increase was found from the age of

frontal cortex was chosen for stereological quantification representing a region with massive and early accumulation of intraneuronal $\mathrm{A} \beta$, with concomitant strong extracellular plaque pathology covering $12.1 \%$ of the frontal cortex at the age of 12 months. To dissect the impact of intraneuronal $\mathrm{A} \beta$ accumulation, the thalamus was chosen as a control region for stereological quantification. It represents a region with no accumulation of intraneuronal $A \beta$ but harbors a comparable amount of extracellular plaques covering $13.1 \%$ of this region at the age of 12 months. Despite the massive amount of plaques, the thalamus was strikingly found to suffer no neuron loss in either 6- or 12month-old APP/PS1KI mice as compared to PS1KI control mice. This is in line with the many negative results reporting no loss of neurons in many mouse models of $\mathrm{AD}$ accumulating various amounts of $A \beta$ plaques $[8,10]$, and altogether suggests that plaques have no significant effect on neuronal survival. In contrast, a neuron loss of about $30 \%$ was found in the frontal cortex already at 6 months of age that did not significantly worsen from 6 to 12 months. Importantly, the only evident pathological difference between the two regions in question was the massive transient accumulation of intraneuronal $A \beta$ in the frontal cortex, which thus must be considered as a major trigger of the neuron loss observed in this region. However, we cannot completely rule out that thalamic neurons differ

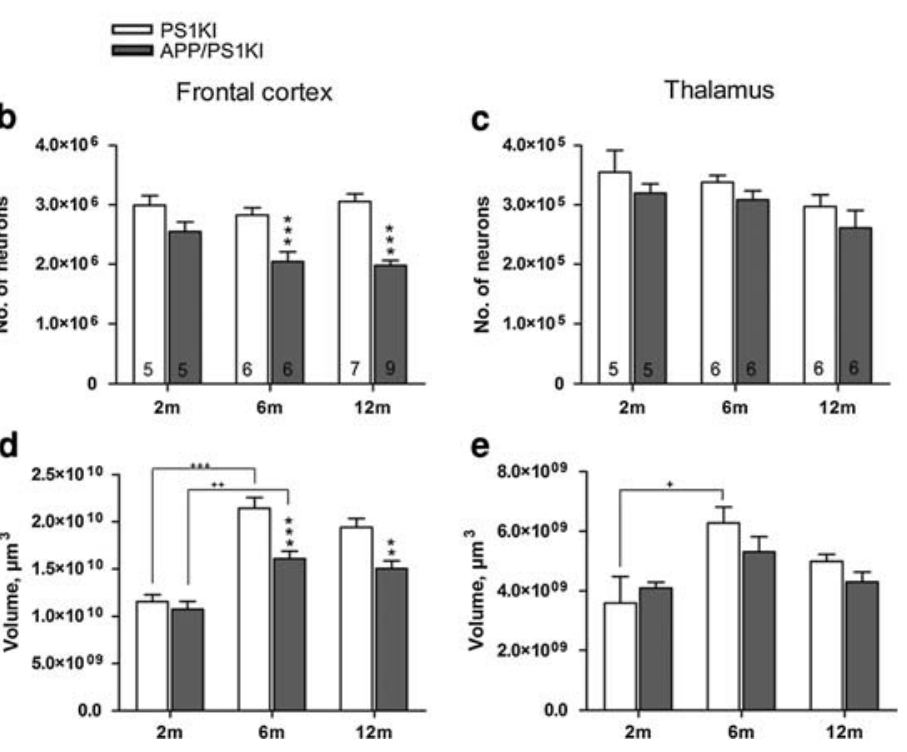

2-6 months for both genotypes owing to normal growth (d). No change in neuron numbers of the thalamus was found between 2-, 6-, and 12-month-old mice (c). As for the cortex, there is an increase in the thalamic volume of PS1KI mice between the age of 2 and 6 months, otherwise no changes in thalamic volume were observed (e). The number of animals analyzed is given directly in the bars (b, c) and was the same for neuron and volume quantifications. All error bars represent mean \pm SEM $* * * P<0.001 ; * * P<0.01 ; * P<0.05$

from cortical neurons in their cellular properties and may therefore be less vulnerable in general. In addition, mutant PS1 may induce toxic effects per se; however, as we have used PS1KI littermates as control mice, which have unchanged neuron numbers during aging in both thalamus and frontal cortex, we conclude that the used PS1-FAD mutations have no effect on neuron survival. Only in combination with APP, A $\beta$-related neurodegeneration is observed.

The transient nature of the intraneuronal accumulation of $A \beta$ adds further support to the hypothesis of its toxicity as significant neuron loss became evident at the age of 6 months; exactly the time point when a decreased intraneuronal $A \beta$ accumulation became obvious. This corresponds to the possibility that the neurons accumulating intracellular $A \beta$ at earlier time points are those lost at a time between the age of 2 and 6 months and correlates with an earlier report claiming that neurons which accumulate $\mathrm{A} \beta$ peptides undergo lysis to give rise to extracellular deposits [6]. However, there are other hypotheses for plaque formation, e.g., compact plaques in the background of high $A \beta 40$ load are suggested to have a different etiology than diffuse plaques with predominant $\mathrm{A} \beta 42$ accumulation, as in the current model. The transient nature of intraneuronal $A \beta$ accumulation preceding massive extracellular plaque pathology has been previously reported in a 
different APP/PS1 transgenic mouse model [14, 28]. The relevance of the finding of an early transient accumulation of $A \beta$ is supported from studies in Down's syndrome (DS) patients who develop $A \beta$ plaques and neurofibrillary tangles as found in $\mathrm{AD}$ patients. Intraneuronal $\mathrm{A} \beta 42$ was found to accumulate only in younger DS patients declining with the development and maturation of plaques $[12,15]$. In addition, another study claims that intraneuronal $A \beta 42$ in human $\mathrm{AD}$ is only detectable in patients with low Braak stages and short disease duration and that the intraneuronal immunoreactivity is lost in patients with more progressive AD [11]. This would correlate with the fact that neurons are not yet lost in pre-clinical AD [23] where intraneuronal accumulation of $\mathrm{A} \beta 42$ would still be detectable; however, with the transition to $\mathrm{AD}$, the intraneuronal immunoreactivity is strongly decreased or undetectable due to the loss of the respective neurons. However, a recent study reported intraneuronal $A \beta$ immunoreactivity throughout the entire lifespan of DS patients and control subjects, leading to the suggestion that this represents a feature of normal neuronal metabolism [20].

The possibility of a transient intraneuronal $A \beta$ accumulation is of great importance for the difficulties of providing proof of the intracellular $A \beta$ hypothesis in human $\mathrm{AD}$ tissue, as this most often represents end stage pathology where adverse neuron loss has already occurred and intraneuronal $A \beta$ therefore may no longer be present.

Altogether, the present study strongly suggests that plaques have no effect on neuronal survival, but that a transient intraneuronal accumulation of $A \beta$ coincides with neuron loss. In our opinion, it is unlikely that soluble extracellular $A \beta$ oligomers cause the observed neurotoxic effects, as they should be present in all plaque-rich areas including both thalamus and the frontal cortex. These findings are in line with a modified $\beta$-amyloid cacscade hypothesis, in which early intraneuronal $A \beta$ accumulation represents the central pathological alteration upstream of extracellular plaque deposition and neuronal dysfunction [27].

Acknowledgments We thank Gerd Multhaup for the generous gift of antibody 23850 and Takaomi C. Saido for the generous gift of antibodies N1D and N3pE. This work was supported by the research program of the Faculty of Medicine, Georg-August-University Göttingen (to O.W.), and the European Commission, Marie Curie Early Stage Training, MEST-CT-2005-020013 (NEURAD), Alzheimer Ph.D. Graduate School.

Conflict of interest statement The authors declare that they have no conflict of interest.

Open Access This article is distributed under the terms of the Creative Commons Attribution Noncommercial License which permits any noncommercial use, distribution, and reproduction in any medium, provided the original author(s) and source are credited.

\section{References}

1. Blanchard V, Moussaoui S, Czech C et al (2003) Time sequence of maturation of dystrophic neurites associated with Abeta deposits in APP/PS1 transgenic mice. Exp Neurol 184:247-263

2. Braak H, Alafuzoff I, Arzberger T, Kretzschmar H, Del Tredici K (2006) Staging of Alzheimer disease-associated neurofibrillary pathology using paraffin sections and immunocytochemistry. Acta Neuropathol 112:389-404

3. Braak H, Braak E (1991) Neuropathological stageing of Alzheimer-related changes. Acta Neuropathol 82:239-259

4. Casas C, Sergeant N, Itier JM et al (2004) Massive CA1/2 neuronal loss with intraneuronal and $\mathrm{N}$-terminal truncated Abeta42 accumulation in a novel Alzheimer transgenic model. Am J Pathol 165:1289-1300

5. Christensen DZ, Bayer TA, Wirths O (2008) Intracellular Abeta triggers neuron loss in the cholinergic system of the APP/PS1KI mouse model of Alzheimer's disease. Neurobiol Aging. doi: 10.1016/j.neurobiolaging.2008.07.022

6. D'Andrea MR, Nagele RG, Wang HY, Peterson PA, Lee DH (2001) Evidence that neurones accumulating amyloid can undergo lysis to form amyloid plaques in Alzheimer's disease. Histopathology 38:120-134

7. Delatour B, Blanchard V, Pradier L, Duyckaerts C (2004) Alzheimer pathology disorganizes cortico-cortical circuitry: direct evidence from a transgenic animal model. Neurobiol Dis 16:4147

8. Duyckaerts C, Potier MC, Delatour B (2008) Alzheimer disease models and human neuropathology: similarities and differences. Acta Neuropathol 115:5-38

9. Fernandez-Vizarra P, Fernandez AP, Castro-Blanco S et al (2004) Intra- and extracellular Abeta and PHF in clinically evaluated cases of Alzheimer's disease. Histol Histopathol 19:823-844

10. Games D, Buttini M, Kobayashi D, Schenk D, Seubert P (2006) Mice as models: transgenic approaches and Alzheimer's disease. J Alzheimers Dis 9:133-149

11. Gouras GK, Tsai J, Naslund J et al (2000) Intraneuronal Abeta42 accumulation in human brain. Am J Pathol 156:15-20

12. Gyure KA, Durham R, Stewart WF, Smialek JE, Troncoso JC (2001) Intraneuronal abeta-amyloid precedes development of amyloid plaques in Down syndrome. Arch Pathol Lab Med 125:489-492

13. Hu L, Wong TP, Cote SL, Bell KF, Cuello AC (2003) The impact of Abeta-plaques on cortical cholinergic and non-cholinergic presynaptic boutons in alzheimer's disease-like transgenic mice. Neuroscience 121:421-432

14. Langui D, Girardot N, El Hachimi KH et al (2004) Subcellular topography of neuronal Abeta peptide in APPxPS1 transgenic mice. Am J Pathol 165:1465-1477

15. Mori C, Spooner ET, Wisniewsk KE et al (2002) Intraneuronal Abeta42 accumulation in Down syndrome brain. Amyloid 9:88102

16. Paxinos G, Franklin KBJ (2001) The mouse brain in stereotaxic coordinates, 2nd edn. Academic Press, New York

17. Schmitz C, Hof PR (2005) Design-based stereology in neuroscience. Neuroscience 130:813-831

18. Simic G, Kostovic I, Winblad B, Bogdanovic N (1997) Volume and number of neurons of the human hippocampal formation in normal aging and Alzheimer's disease. J Comp Neurol 379:482-494

19. Tsai J, Grutzendler J, Duff K, Gan WB (2004) Fibrillar amyloid deposition leads to local synaptic abnormalities and breakage of neuronal branches. Nat Neurosci 7:1181-1183

20. Wegiel J, Kuchna I, Nowicki K et al (2007) Intraneuronal Abeta immunoreactivity is not a predictor of brain amyloidosis-beta or neurofibrillary degeneration. Acta Neuropathol 113:389-402 
21. West MJ (2002) Design-based stereological methods for counting neurons. Prog Brain Res 135:43-51

22. West MJ, Coleman PD, Flood DG, Troncoso JC (1994) Differences in the pattern of hippocampal neuronal loss in normal ageing and Alzheimer's disease. Lancet 344:769-772

23. West MJ, Kawas CH, Stewart WF, Rudow GL, Troncoso JC (2004) Hippocampal neurons in pre-clinical Alzheimer's disease. Neurobiol Aging 25:1205-1212

24. West MJ, Slomianka L, Gundersen HJ (1991) Unbiased stereological estimation of the total number of neurons in the subdivisions of the rat hippocampus using the optical fractionator. Anat Rec 231:482-497

25. Whitehouse PJ, Price DL, Clark AW, Coyle JT, DeLong MR (1981) Alzheimer disease: evidence for selective loss of cholinergic neurons in the nucleus basalis. Ann Neurol 10:122-126
26. Wirths O, Breyhan H, Schafer S, Roth C, Bayer TA (2008) Deficits in working memory and motor performance in the APP/ PS1ki mouse model for Alzheimer's disease. Neurobiol Aging 29:891-901

27. Wirths O, Multhaup G, Bayer TA (2004) A modified beta-amyloid hypothesis: intraneuronal accumulation of the beta-amyloid peptide-the first step of a fatal cascade. J Neurochem 91:513520

28. Wirths O, Multhaup G, Czech C et al (2002) Intraneuronal APP/ A beta trafficking and plaque formation in beta-amyloid precursor protein and presenilin-1 transgenic mice. Brain Pathol 12:275286

29. Wirths O, Weis J, Kayed R, Saido TC, Bayer TA (2007) Agedependent axonal degeneration in an Alzheimer mouse model. Neurobiol Aging 28:1689-1699 